\title{
Optimal Parameters of Service in a Public Transportation Market with Pricing
}

\author{
Hongwei Gao $\mathbb{D}^{1},{ }^{1}$ Vladimir V. Mazalov $\mathbb{D D}^{2}$ and Juan Xue ${ }^{1}$ \\ ${ }^{1}$ School of Mathematics and Statistics, Qingdao University and Institute of Applied Mathematics of Shandong, \\ Qingdao 266071, China \\ ${ }^{2}$ Institute of Applied Mathematical Research, Karelian Research Center, Russian Academy of Sciences, Pushkinskaya 11, \\ Petrozavodsk 185910, Russia \\ Correspondence should be addressed to Vladimir V. Mazalov; vmazalov@krc.karelia.ru
}

Received 5 December 2019; Accepted 12 February 2020; Published 10 March 2020

Guest Editor: Young-Ji Byon

Copyright (C) 2020 Hongwei Gao et al. This is an open access article distributed under the Creative Commons Attribution License, which permits unrestricted use, distribution, and reproduction in any medium, provided the original work is properly cited.

\begin{abstract}
The problem of choosing the optimal parameters of service by carriers in public transport passenger flows is considered. This problem is modeled as a two-stage game. At the first stage, the players (carriers) select the parameters of their service (the number and schedule of vehicles, etc.). At the second stage, the players announce the prices of service and the consumers choose an appropriate service. The Wardrop equilibrium is applied to the competition model with rational consumers preferring the carriers with minimum cost, where the cost of service includes the price and also the expected trip time. The equilibrium in the pricing game is found and the optimal parameters of service as a solution to a noncooperative game are determined. Some results of computer simulations are presented.
\end{abstract}

\section{Introduction}

Traffic control is the subject of many studies. Since the 1950s, models with different optimality principles and corresponding numerical methods have been used in this area of research. In 1952, Wardrop hypothesized that any transport system reaches equilibrium after a certain period of time and also formulated two principles of an equilibrium distribution of traffic flows [1]. In accordance with the Wardrop principles, the trip time on all existing routes is the same for all road users and less than the trip time of any road user deviating from his route; moreover, the average trip time is minimized. At present, the concept of Wardrop equilibrium is the main tool in the theory of transport flows $[2,3]$.

In this paper, we investigate the problem of equilibrium traffic flows for passengers of urban public transport, which includes buses, trolleybuses, trams, subway, taxis, and bicycles.

In the Wardrop approach, the cost of a transport service for users (passengers) is the trip time when using a corresponding carrier. This principle can be generalized by considering the costs composed of the trip time and also the total costs of road users on all routes. Similar to the Wardrop model, users are assumed to be rational agents who compare the costs from using a particular service and choose a service with the lowest costs. The cost function may include the price of a service, the average trip time, risks, and other relevant factors.

As an illustration to this problem, the choice of routes around the city of Qingdao can be considered, see Figure 1. At the end of lectures, students of Qingdao University (point A) move to the Qingdao railway station (point B). They can choose one of the three bus routes shown in the diagram. All buses have different schedules and different ticket prices. Being guided by these parameters, students are distributing themselves among the routes in accordance with the Wardrop principle or its generalization.

In [4], this generalization was made for the cost function that includes the price of service plus the trip time spent. Equations for calculating the equilibrium in this model were obtained using the Karush-Kuhn-Tucker theorem.

The behavior of users was taken into account in a number of other research works as well. For example, in [5], part of the users was considered to be oblivious; while the 


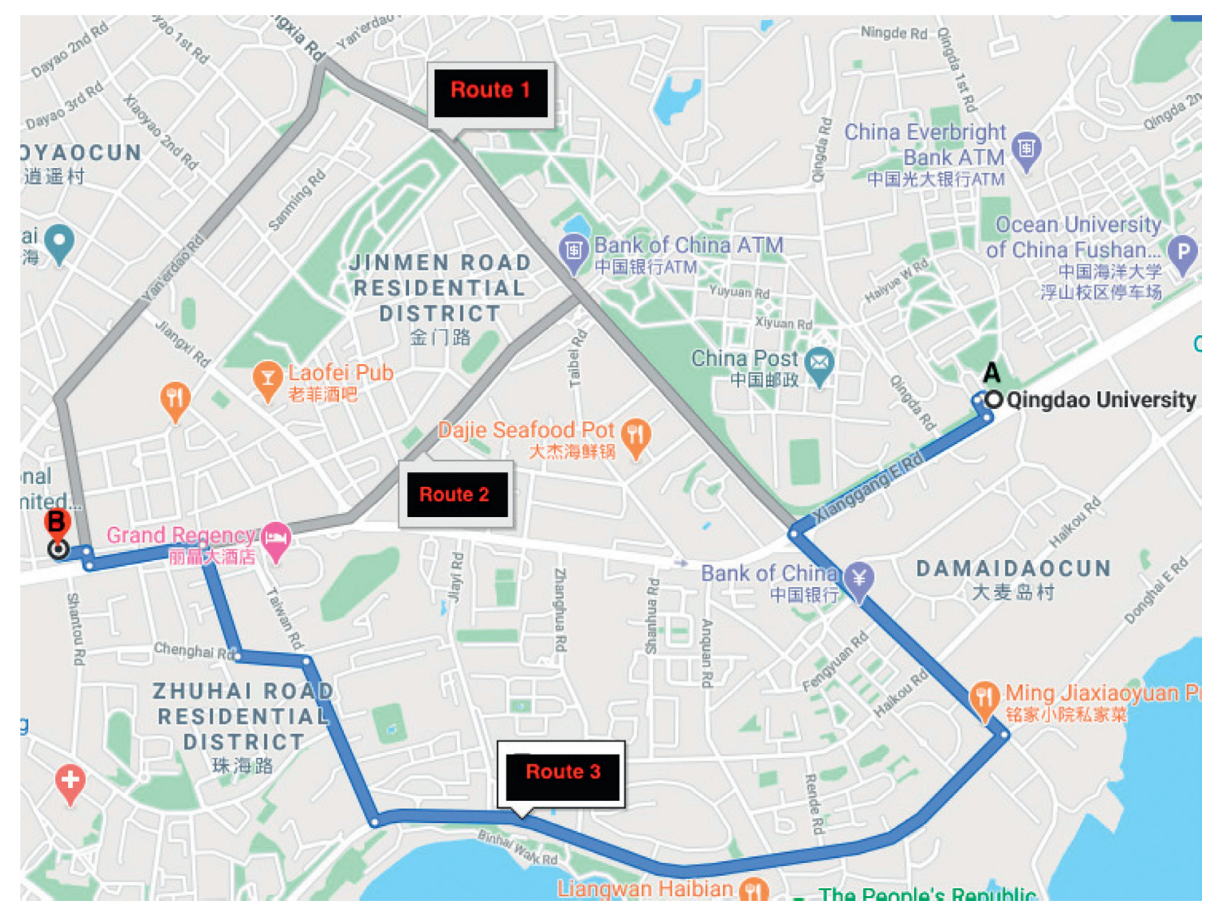

Figure 1: Routes in city of Qingdao.

rational users follow a strategy that minimizes their individual cost, an oblivious user prefers cheapest routes.

It is also important to consider the case in which users can choose a free mode of transportation, such as bicycles or motorcycles. Now this way of transportation is given a lot of attention [6]. In addition, many local authorities subsidize public transport, and then tickets for some municipal transport become much cheaper than for commercial transport. This can greatly affect the equilibrium in the distribution of passenger flows.

Note that the type of latency functions for public transport can be selected in different ways. Some publications followed this approach within the framework of queueing theory, see [7-11].

For a transport flow of intensity $\lambda$, the cited authors defined the latency as the average service time $1 /(\mu-\lambda)$, i.e., as the expected sojourn time of a user in a queueing system $M / M / n$. In paper [11], the Wardrop principle was applied to networks of general topology and the BPR (Bureau of Public Road) latency functions [12].

The efficiency of a city traffic control system can be evaluated through the price of anarchy, initially introduced by Koutsopias and Papadimitriou [13]. This is the ratio of the social costs of the traffic system in the competitive and cooperative cases. The price of anarchy was evaluated for different classes of latency functions. Roughgarden showed that the price of anarchy is exactly $4 / 3$ in the case of linear latency functions [11]. For the polynomial latency functions of maximum degree $d$, Roughgarden [11] established that the price of anarchy is $(d+1)^{1+1 / d} /\left((d+1)^{1+1 / d}-d\right)$. The price of anarchy was also studied for latency functions that involve a delay function of $M / M / 1$ queues $[8,11,14]$. These results were obtained for the Wardrop model, where the consumers minimize the expected trip time. For a generalized case in which the costs include the trip time and price of service, in paper [15] it was demonstrated that the price of anarchy can be infinite. The influence of oblivious users on the price of anarchy was analyzed in [5].

In addition to the problem of finding equilibrium flows in a transport system, for each carrier, it is important to determine the optimal parameters of passenger service, such as schedule, the number, type and size of vehicles, and the time interval and speed of vehicles.

In the following, we formulate this problem as a two-stage game-theoretic model. At the first stage, the players choose the parameters of their service. At the second stage, the players announce the prices of service and the consumers select an appropriate service. We consider rational consumers for whom the cost of service includes the price and trip time. The Wardrop equilibrium is applied to determine the optimal flow in the network. Since the payoffs of the players are known, we return to the first stage and find the optimal parameters of service as the equilibrium in the new game.

This game can be considered as a variant of the multileader multifollower Stackelberg game [16, 17]. In such games, multiple Stackelberg leaders compete in a noncooperative game following which followers play a noncooperative game amongst themselves, taking the decisions of leaders as fixed. Under this approach, each player in our transportation game can be represented by two agents, a leader, and a follower. The leading agent is responsible for the service parameters, which may include the number of transport units and schedule, and the follower is responsible for the service prices.

The paper is organized in the following way. In Section 2, we give a formalization of the two-stage transportation game 
with pricing. In Section 3, we construct equilibrium in the two-player game on two parallel routes with the linear latency function. In Section 4, we introduce an externality into this model and calculate equilibrium in the two-stage game. In Section 5, we find equilibrium for a transportation system with a possible use of service free of charge. We analyze this model for the latency function of the form $M / M / 1$. In Section 6, we outline further research.

\section{Two-Stage Game}

Consider a two-stage noncooperative nonzero-sum game of $n$ players, which is associated with a queueing system that operates on a transportation network. Denote a transportation $n$ player game by $\Gamma=\left\langle N, G,\left\{Z_{i}\right\}_{\{i \in N\}},\left\{H_{i}\right\}_{\{i \in N\}}\right\rangle$, where $N=\{1, \ldots, n\}$ means the set of players (carriers) serving passengers on a graph $G=\langle V, E\rangle$ with a node set $V(|V|=m)$ and an edge set $E$. For each player $i$, there exists a set of routes $Z_{i}$ from a starting node $v_{s} \in V$ to a destination node $v_{t} \in V$ served by player $i$. Therefore, $Z_{i}=$ $\left\{R_{s t}: v_{s}, v_{t} \in V\right\}, i=1, \ldots, n$. Each route represents a path, i.e., a sequence of nodes connected by edges $R_{s t}=$ $\left(v_{s}, v_{s+1}, \ldots, v_{t}\right)$ so that the end of one edge is the beginning of another edge $\left(v_{s}, v_{s+1}\right), \ldots,\left(v_{t-1}, v_{t}\right) \in E$.

We assume that the passenger flows over this graph are Poisson processes of some intensity. We introduce the matrix of flow intensities $\left\{\lambda_{s t}\right\}$ from node $v_{s}$ to node $v_{t}$ for different $s, t=1, \ldots, m$.

The game is organized as follows. At the first stage, the players determine the parameters of service on each route, such as schedule, the number and size of vehicles, and the time interval and speed of vehicles. Denote these control parameters by $q^{i}$ for each player $i=1, \ldots, n$.

Thus, at the first stage, each player $i$ determines the control parameters $q_{s t}^{i}, R_{s t} \in Z_{i}$, and a strategy profile $\left\{q^{i}, i \in N\right\}$ is formed accordingly.

At the second stage, each player $i$ establishes prices $p_{i}^{R}$ of its service on each route $R \in Z_{i}$. As a result, a strategy profile $p^{Z}=\left\{p_{i}^{R}, R \in Z_{i}, i=1, \ldots, n\right\}$ is formed. We assume that the passengers minimize their expected costs, which include the price of service and also the expected sojourn time (trip time). The expected trip time is a nondecreasing function of traffic intensity that takes the form $f_{s t}(\lambda)$ on each route $R_{s t}$. The latency function $f_{s t}\left(\lambda_{e}\right)$ can be described by $1 /\left(\mu_{s t}-\lambda_{s t}\right)$ (in accordance with queueing theory) [9-11] or can have the linear $t_{s t}\left(1+\alpha_{s t} \lambda_{s t} / c_{s t}\right)$ or polynomial $t_{s t}\left(1+\alpha_{s t}\left(\lambda_{s t} / c_{s t}\right)^{\beta}\right)$ forms (the well-known BPR latency function) [11]. Here, $t_{s t}$ is the trip time on an unoccupied route; $c_{s t}$ specifies the capacity of a route; the constants $\alpha_{s t}$ and $\beta_{s t}$ capture routespecific features that may affect the impact of the flow-tocapacity ratio on the trip time.

Denote by $u(p, t)$ the costs of passengers who used a service with a ticket price $p$ and a trip time $t$. In papers $[4,15]$, the costs were expressed as the ticket price plus the expected trip time, and the customers were assumed to choose the cheapest service. In this case, the incoming Poisson flow of intensity $\lambda_{s t}$ is decomposed into $n$ subflows of intensities $\lambda_{s t}^{i}$, where $\sum_{i=1}^{n} \lambda_{s t}^{i}=\lambda_{s t}$. Note that $\lambda_{s t}^{i}=0$ if the set $Z_{i}$ of player $i$ has no path $R_{s t}$.
The costs of a passenger preferring service $i$ on some route $R_{s t} \in Z_{i}$ include the price of the service and also the expected trip time:

$$
u\left(p_{s t}^{i}, \lambda_{s t}^{i}\right) .
$$

Following the Wardrop principle, we assume that the equilibrium costs of all passengers on competing directions coincide for all services. This feature allows evaluating the intensities $\lambda_{s t}^{i}$ for all services $i=1, \ldots, n$ and routes $R_{s t}$. That is,

$$
u\left(p_{s t}^{i}, \lambda_{s t}^{i}\right)=u\left(p_{s t}^{j}, \lambda_{s t}^{j}\right),
$$

for all $i, j$ such that $R_{s t} \in Z_{i}$ and $R_{s t} \in Z_{j}$. If the price of some service on a route turns out to be too high, the passenger flow is distributed among other services, and the former service does not compete. In other words, equilibrium prices should be found among balanced prices.

The payoff of player $i \in N$ can be defined by its income per unit time from serving all flows on all routes of this player minus the operating costs, i.e.,

$$
H_{i}\left(p_{i}^{Z}\right)=\sum_{(s t): R_{s t} \in Z_{i}} \lambda_{s t}^{i} p_{s t}^{i}-C_{i}\left(q^{i}\right), \quad i \in N .
$$

Thus, we have a two-stage game of $n$ players. First, we fix the parameters of service $\left\{q^{i}, i \in N\right\}$. Next, we find a Nash equilibrium in the pricing game with the payoff function (1), where the cost $C_{i}\left(q^{i}\right)$ is excluded. Let $\left\{p_{i}^{*}, i \in N\right\}$ be the equilibrium in the pricing game. The prices and flows in the equilibrium will depend on the service parameters $q=$ $\left\{q^{i}, i \in N\right\}$.

Then, we return to the first stage and find a Nash equilibrium in the game with payoffs (1) among the strategies $q=\left\{q^{i}, i \in N\right\}$.

\section{Game of Two Players on Two Parallel Routes with Linear Latency Function}

Our analysis begins with the transportation game of two carriers rendering their services between two points $v_{1}$ and $v_{2}$. We assume that carriers 1 and 2 (players 1 and 2) are operating on two parallel routes.

Let the service time of player $i$ have the linear BPR latency function:

$$
t_{i}\left(1+\alpha_{i}\left(\frac{\lambda_{i}}{c_{i}}\right)\right)
$$

where $i=1,2$. For player $i$, the parameter $t_{i}$ corresponds to the speed along a route, $c_{i}$ is a capacity of the route, and $\alpha$ is some parameter. At the second stage, we fix the parameters $q^{1}=\left(t_{1}, c_{1}, \alpha_{1}\right)$ and $q^{2}=\left(t_{2}, c_{2}, \alpha_{2}\right)$, but we will return to the problem of their choice later.

The incoming flow is a Poisson process with an intensity $\lambda$. At the second stage, the players announce the prices of service, $p_{1}$ and $p_{2}$, respectively. The passengers select the minimum-cost service. As a result, the incoming flow is decomposed into two subflows with intensities $\lambda_{1}$ and $\lambda_{2}$, where $\lambda_{1}+\lambda_{2}=\lambda$. 
The costs of passengers include the price of service and the expected trip time. We assume that the costs are a convex combination of these criteria:

$$
u(p, \lambda)=k p+(1-k) t\left(1+\alpha\left(\frac{\lambda}{c}\right)\right)
$$

where $k: 0 \leq k \leq 1$. Because the parameters $k, \alpha$, and $c$ are constant, without loss of generality we may simplify the expression of the costs:

$$
u(p, \lambda)=p+t\left(1+\frac{\lambda}{c}\right) .
$$

In accordance with the Wardrop principle, in an equilibrium the intensities of subflows $\lambda_{1}$ and $\lambda_{2}$ satisfy the balance equation:

$$
\begin{aligned}
u_{1}\left(p_{1}, p_{2}, \lambda_{1}, \lambda_{2}\right) & =u_{2}\left(p_{1}, p_{2}, \lambda_{1}, \lambda_{2}\right) \\
\operatorname{or} p_{1}+t_{1}\left(1+\frac{\lambda_{1}}{c_{1}}\right) & =p_{2}+t_{2}\left(1+\frac{\lambda_{2}}{c_{2}}\right) \\
\lambda_{1}+\lambda_{2} & =\lambda .
\end{aligned}
$$

From these equations, we obtain

$$
\begin{aligned}
& \lambda_{1}=\frac{p_{2}-p_{1}+t_{2}-t_{1}+\left(t_{2} / c_{2}\right) \lambda}{\left(t_{1} / c_{1}\right)+\left(t_{2} / c_{2}\right)}, \\
& \lambda_{2}=\frac{p_{1}-p_{2}+t_{1}-t_{2}+\left(t_{1} / c_{1}\right) \lambda}{\left(t_{1} / c_{1}\right)+\left(t_{2} / c_{2}\right)} .
\end{aligned}
$$

Consider the pricing game with the payoffs:

$$
\begin{aligned}
& h_{1}\left(p_{1}, p_{2}\right)=\lambda_{1} p_{1}, \\
& h_{2}\left(p_{1}, p_{2}\right)=\lambda_{2} p_{2} .
\end{aligned}
$$

This is a convex game with the equilibrium:

$$
\begin{aligned}
& p_{1}^{*}=\frac{1}{3}\left(t_{2}-t_{1}+\lambda\left(\frac{t_{1}}{c_{1}}+2 \frac{t_{2}}{c_{2}}\right)\right), \\
& p_{2}^{*}=\frac{1}{3}\left(t_{1}-t_{2}+\lambda\left(2 \frac{t_{1}}{c_{1}}+\frac{t_{2}}{c_{2}}\right)\right) .
\end{aligned}
$$

The intensities of subflows in the equilibrium are

$$
\begin{aligned}
& \lambda_{1}^{*}=\frac{t_{2}-t_{1}+\lambda\left(t_{1} / c_{1}+2 t_{2} / c_{2}\right)}{3\left(t_{1} / c_{1}+t_{2} / c_{2}\right)}, \\
& \lambda_{2}^{*}=\frac{t_{1}-t_{2}+\lambda\left(2 t_{1} / c_{1}+t_{2} / c_{2}\right)}{3\left(t_{1} / c_{1}+t_{2} / c_{2}\right)} .
\end{aligned}
$$

Now, we return to the first stage of the game. The equilibrium payoffs (2) of players, $H_{1}\left(p_{1}^{*}, p_{2}^{*}\right)$ and $H_{2}\left(p_{1}^{*}, p_{2}^{*}\right)$, depend on the parameters of service, $q_{1}=\left(t_{1}, c_{1}\right)$ and $q_{2}=\left(t_{2}, c_{2}\right)$. Choosing specific values of these parameters, we may adjust the costs. This leads to a new game with the payoff functions:

$$
\begin{aligned}
& H_{1}\left(q_{1}, q_{2}\right)=\frac{\left(t_{2}-t_{1}+\lambda\left(t_{1} / c_{1}+2 t_{2} / c_{2}\right)\right)^{2}}{9\left(t_{1} / c_{1}+t_{2} / c_{2}\right)}-C_{1}\left(t_{1}, c_{1}\right), \\
& H_{2}\left(q_{1}, q_{2}\right)=\frac{\left(t_{1}-t_{2}+\lambda\left(2 t_{1} / c_{1}+t_{2} / c_{2}\right)\right)^{2}}{9\left(t_{1} / c_{1}+t_{2} / c_{2}\right)}-C_{2}\left(t_{2}, c_{2}\right),
\end{aligned}
$$

where $C_{1}$ and $C_{2}$ are the operating costs of players for these parameters of service.

3.1. Computer Simulations. In this section, the results of computer simulations in which we find the equilibrium parameters $\left(t_{1}^{*}, t_{2}^{*}\right)$ for the cost functions $C_{1}\left(t_{1}\right)=r_{1} / t_{1}^{2}$ and $C_{2}\left(t_{2}\right)=r_{2} / t_{2}^{2}$ are presented.

Table 1 shows the equilibrium parameters $\left(t_{1}^{*}, t_{2}^{*}\right)$ calculated for the fixed values $c_{1}=c_{2}=1$ and the costs $C_{1}\left(t_{1}\right)=r_{1} / t_{1}^{2}, C_{2}\left(t_{2}\right)=r_{2} / t_{2}^{2}$, where $r_{1}=1$ and $r_{2}=1.5$.

In this case, the capacities of both routes are equal and the only difference concerns the costs. Since $r_{1}<r_{2}$, the second service is more expensive.

Clearly, if the incoming flow is increasing, the prices and the payoffs in the equilibrium are also increasing. However, there is some irregularity in the behavior of equilibrium operating times (speeds) of services as follows. Under increasing the incoming flow, the equilibrium operating time $t_{1}$ of the first service is decreasing; however, the equilibrium operating time $t_{2}$ of the second service is first decreasing and then, after some instant, starts increasing.

The second service is more expensive; therefore, the equilibrium payoff of the first service is higher than that of the second service.

\section{Game of Two Players with Externalities}

Consider a transportation network composed of two parallel routes with the linear latency functions:

$$
\begin{aligned}
& f_{1}(\lambda)=t_{1}\left(1+a_{1} \lambda_{1}+b_{1} \lambda_{2}\right), \\
& f_{2}(\lambda)=t_{1}\left(1+a_{2} \lambda_{2}+b_{2} \lambda_{1}\right) .
\end{aligned}
$$

Two carriers (players) are serving consumers on the two parallel routes with prices $p_{1}$ and $p_{2}$, respectively. The incoming flow $\lambda$ is decomposed into two subflows $\lambda_{1}$ and $\lambda_{2}$, $\lambda_{1}+\lambda_{2}=\lambda$, on routes 1 and 2 , respectively. We assume that the flow $\lambda$ is sufficiently large that all routes are used by the consumers.

Here, the parameters $a_{1}$ and $a_{2}$ describe an internal effect of the subflows $\lambda_{1}$ and $\lambda_{2}$ on routes 1 and 2 , respectively. By analogy, the parameters $b_{1}$ and $b_{2}$ describe an external effect of the subflows $\lambda_{2}$ and $\lambda_{1}$ on routes 1 and 2 .

We assume that $a_{i}>b_{i}, b_{j}, i \neq j$; the internal effect is more important than the external one for traffic control. However, the external parameters may significantly affect the traffic delays and can be a goal of improvement in a transportation system.

Following the same considerations as in Section 3, we obtain the equilibrium prices and flows in the pricing game. In accordance with the Wardrop principle, in equilibrium, 
TABLE 1: Optimal parameters of services in equilibrium.

\begin{tabular}{lcccccccc}
\hline$\lambda$ & $t_{1}$ & $t_{2}$ & $p_{1}^{*}$ & $p_{2}^{*}$ & $\lambda_{1}^{*}$ & $\lambda_{2}^{*}$ & $H_{1}^{*}$ & $H_{2}^{*}$ \\
\hline 1 & 1.772 & 2.656 & 2.656 & 1.772 & 0.6 & 0.4 & 1.274 & 0.496 \\
1.1 & 1.732 & 2.610 & 2.841 & 1.934 & 0.654 & 0.445 & 1.526 & 0.642 \\
1.2 & 1.696 & 2.582 & 3.039 & 2.094 & 0.710 & 0.489 & 1.811 & 0.800 \\
1.3 & 1.660 & 2.572 & 3.252 & 2.249 & 0.768 & 0.531 & 2.138 & 0.969 \\
1.4 & 1.624 & 2.587 & 3.493 & 2.402 & 0.829 & 0.570 & 2.519 & 1.146 \\
1.5 & 1.585 & 2.632 & 3.773 & 2.552 & 0.894 & 0.605 & 2.978 & 1.327 \\
\hline
\end{tabular}

the intensities of the subflows $\lambda_{1}$ and $\lambda_{2}$ satisfy the balance equation $p_{1}+f_{1}(\lambda)=p_{2}+f_{2}(\lambda)$. This yields

$$
\begin{aligned}
& \lambda_{1}=\frac{p_{2}-p_{1}+t_{2}-t_{1}+\left(a_{2} t_{2}-b_{1} t_{1}\right) \lambda}{\Delta}, \\
& \lambda_{2}=\frac{p_{1}-p_{2}+t_{1}-t_{2}+\left(a_{1} t_{1}-b_{2} t_{2}\right) \lambda}{\Delta},
\end{aligned}
$$

where $\Delta=a_{1} t_{1}+a_{2} t_{2}-b_{1} t_{1}-b_{2} t_{2}$.

For the pricing game with the payoffs,

$$
\begin{aligned}
& h_{1}\left(p_{1}, p_{2}\right)=\lambda_{1} p_{1}, \\
& h_{2}\left(p_{1}, p_{2}\right)=\lambda_{2} p_{2},
\end{aligned}
$$

we find equilibrium

$$
\begin{aligned}
& p_{1}^{*}=\frac{1}{3}\left(t_{2}-t_{1}+\lambda\left(a_{1} t_{1}-b_{2} t_{2}+2\left(a_{2} t_{2}-b_{1} t_{1}\right)\right)\right), \\
& p_{2}^{*}=\frac{1}{3}\left(t_{1}-t_{2}+\lambda\left(2\left(a_{1} t_{1}-b_{2} t_{2}\right)+a_{2} t_{2}-b_{1} t_{1}\right)\right) .
\end{aligned}
$$

The intensities of subflows in the equilibrium are

$$
\begin{aligned}
& \lambda_{1}^{*}=\frac{p_{1}^{*}}{\Delta}, \\
& \lambda_{2}^{*}=\frac{p_{2}^{*}}{\Delta} .
\end{aligned}
$$

Now, consider the game at the first stage. The equilibrium payoffs of the players, $H_{1}\left(p_{1}^{*}, p_{2}^{*}\right)$ and $H_{2}\left(p_{1}^{*}, p_{2}^{*}\right)$, depend on the parameters $q_{1}=\left(t_{1}, a_{1}, b_{1}\right)$ and $q_{2}=\left(t_{2}, a_{2}, b_{2}\right)$ of the latency functions. Changing these parameters, the players can improve the transportation system. However, this procedure can be rather expensive.

Let the players be interested in reducing the external influence on their own traffic. In other words, player 1 seeks to minimize $b_{1}$ while player 2 seeks to minimize $b_{2}$. In this case, we arrive in the game with the payoff functions:

$$
\begin{aligned}
& H_{1}\left(b_{1}, b_{2}\right)=\frac{\left(t_{2}-t_{1}+\lambda\left(a_{1} t_{1}-b_{2} t_{2}+2\left(a_{2} t_{2}-b_{1} t_{1}\right)\right)\right)^{2}}{9 \Delta}-C_{1}\left(b_{1}\right), \\
& H_{2}\left(b_{1}, b_{2}\right)=\frac{\left(t_{1}-t_{2}+\lambda\left(2\left(a_{1} t_{1}-b_{2} t_{2}\right)+a_{2} t_{2}-b_{1} t_{1}\right)\right)^{2}}{9 \Delta}-C_{2}\left(b_{2}\right),
\end{aligned}
$$

where $C_{1}$ and $C_{2}$ the operating costs of players for these parameters of service.
4.1. Computer Simulations. We assume that the latency functions have form

$$
\begin{aligned}
& f_{1}(\lambda)=2\left(1+3 \lambda_{1}+b_{1} \lambda_{2}\right), \\
& f_{2}(\lambda)=3\left(1+2 \lambda_{2}+b_{2} \lambda_{1}\right) .
\end{aligned}
$$

Hence, the first route is faster than the second one for small traffic, but the latency is increasing more intensively for large traffic.

Then, the equilibrium prices are

$$
\begin{aligned}
& p_{1}^{*}=\frac{1}{3}\left(1+\lambda\left(18-3 b_{2}-4 b_{1}\right)\right), \\
& p_{2}^{*}=\frac{1}{3}\left(-1+\lambda\left(18-6 b_{2}-2 b_{1}\right)\right) .
\end{aligned}
$$

As a result, the payoff functions in this game are given by

$$
\begin{aligned}
& H_{1}\left(b_{1}, b_{2}\right)=\frac{\left(1+\lambda\left(18-3 b_{2}-4 b_{1}\right)\right)^{2}}{9\left(12-2 b_{1}-3 b_{2}\right)}-C_{1}\left(t_{1}, a_{1}, b_{1}\right), \\
& H_{2}\left(b_{1}, b_{2}\right)=\frac{\left(1+\lambda\left(18-6 b_{2}-2 b_{1}\right)\right)^{2}}{9\left(12-2 b_{1}-3 b_{2}\right)}-C_{2}\left(t_{2}, a_{2}, b_{2}\right) .
\end{aligned}
$$

For example, if the cost functions have the form $C_{i}\left(b_{i}\right)=$ $c_{i} / b_{i}, i=1,2$ and the intensity of traffic is $\lambda=1$, we obtain the following equilibrium values in this game, see Table 2 .

Clearly, increasing the cost yields gives higher equilibrium values for $b_{1}$ and $b_{2}$. At the same time, the prices of both services (and hence the payoffs) are decreasing accordingly. Also, we may conclude that the parameters $t_{i}, i=$ 1,2 are more important for the payoffs than $a_{i}, i=1,2$. Here, $t_{1}<t_{2}$ and $a_{1}>a_{2}$, but in equilibrium, we obtain $H_{1}>H_{2}$.

\section{Transportation Game with Possible Use of Service Free of Charge}

Our analysis proceeds to the transportation game with externality in which two carriers are rendering their services between two points $v_{1}$ and $v_{2}$, and, in addition, the consumers can use a cheap transportation service with a fixed price $p_{0}$.

It can be a free-of-charge way of personal transportation, e.g., biking (which matches the case $p_{0}=0$ ). Also, in some cities, the local government is supporting public transport and the price of bus tickets is sufficiently small.

A usual way of transportation in a city is presented in Figure 2. A person who has to travel from a starting point $v_{1}$ to a destination point $v_{2}$ can choose bike or public transport (bus or taxi). Both means of public transport are faster than bike but costly. Thus, the choice depends on personal preferences.

We assume that the service time of player $i$ has the exponential probability density function with an intensity $\mu_{i}$ on the route $\left(v_{1}, v_{2}\right), i=1,2$. These parameters form a strategy profile $\left(q^{1}, q^{2}\right)$ of the players at the first stage of the game. 
TABle 2: Optimal parameters of services in equilibrium.

\begin{tabular}{ccccc}
\hline & $c_{2}=0.03$ & $c_{2}=0.06$ & $c_{2}=0.09$ & $c_{2}=0.12$ \\
\hline & $b_{1}=0.187$ & $b_{1}=0.187$ & $b_{1}=0.187$ & $b_{1}=0.187$ \\
& $b_{2}=0.157$ & $b_{2}=0.223$ & $b_{2}=0.273$ & $b_{2}=0.316$ \\
$c_{1}=0.03$ & $p_{1}=5.925$ & $p_{1}=5.861$ & $p_{1}=5.811$ & $p_{1}=5.768$ \\
& $p_{2}=5.227$ & $p_{2}=5.096$ & $p_{2}=4.996$ & $p_{2}=4.910$ \\
& $H_{1}=2.988$ & $H_{1}=2.974$ & $H_{1}=2.964$ & $H_{1}=2.955$ \\
& $H_{2}=2.259$ & $H_{2}=2.101$ & $H_{2}=1.979$ & $H_{2}=1.877$ \\
\hline \multirow{5}{c}{$=0.06$} & $b_{1}=0.265$ & $b_{1}=0.265$ & $b_{1}=0.265$ & $b_{1}=0.265$ \\
& $b_{2}=0.157$ & $b_{2}=0.222$ & $b_{2}=0.273$ & $b_{2}=0.315$ \\
& $p_{1}=5.823$ & $p_{1}=5.758$ & $p_{1}=5.707$ & $p_{1}=5.665$ \\
& $p_{2}=5.176$ & $p_{2}=5.046$ & $p_{2}=4.944$ & $p_{2}=4.859$ \\
& $H_{1}=2.856$ & $H_{1}=2.842$ & $H_{1}=2.831$ & $H_{1}=2.822$ \\
& $H_{2}=2.444$ & $H_{2}=2.086$ & $H_{2}=1.965$ & $H_{2}=1.863$ \\
\hline$c_{1}=0.09$ & $b_{1}=0.326$ & $b_{1}=0.325$ & $b_{1}=0.325$ & $b_{1}=0.324$ \\
& $b_{2}=0.157$ & $b_{2}=0.222$ & $b_{2}=0.273$ & $b_{2}=0.315$ \\
& $p_{1}=5.742$ & $p_{1}=5.678$ & $p_{1}=5.627$ & $p_{1}=5.586$ \\
& $p_{2}=5.135$ & $p_{2}=5.006$ & $p_{2}=4.904$ & $p_{2}=4.820$ \\
& $H_{1}=2.754$ & $H_{1}=2.740$ & $H_{1}=2.729$ & $H_{1}=2.720$ \\
& $H_{2}=2.233$ & $H_{2}=2.075$ & $H_{2}=1.953$ & $H_{2}=1.852$ \\
\hline$b_{1}=0.376$ & $b_{1}=0.375$ & $b_{1}=0.375$ & $b_{1}=0.375$ \\
$b_{2}=0.157$ & $b_{2}=0.222$ & $b_{2}=0.272$ & $b_{2}=0.315$ \\
$c_{1}=0.12$ & $p_{1}=5.675$ & $p_{1}=5.611$ & $p_{1}=5.561$ & $p_{1}=5.518$ \\
$p_{2}=5.102$ & $p_{2}=4.972$ & $p_{2}=4.872$ & $p_{2}=4.786$ \\
$H_{1}=2.669$ & $H_{1}=2.654$ & $H_{1}=2.644$ & $H_{1}=2.635$ \\
$H_{2}=2.224$ & $H_{2}=2.066$ & $H_{2}=1.944$ & $H_{2}=1.842$ \\
\hline & & &
\end{tabular}

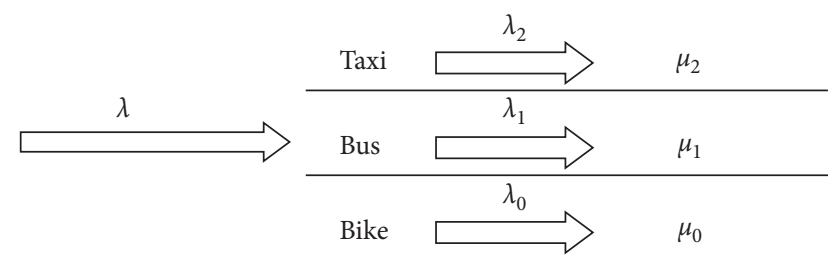

Figure 2: Scheme of public urban transport.

The incoming flow is a Poisson process with an intensity $\lambda$. In accordance with queueing theory, for the Poisson flow $\lambda$ and a traffic flow $\mu$ on the route $\left(v_{1}, v_{2}\right)$, the expected trip time is given by $1 /(\mu-\lambda)$. Consequently, for a consumer selecting the service $\mu_{1}$ or $\mu_{2}$ (or deciding to use bike, $\mu_{0}$ ), the expected trip time is $\mu_{j}-\lambda_{j}$, where $\lambda_{j}$ is a flow in this route $j=0,1,2$.

We assume that the parameters $\mu_{i}, i=0,1,2$ are fixed and consider the second stage of the game. At the second stage, players 1 and 2 announce the prices of service, $p_{1}$ and $p_{2}$, respectively. Let the passengers be rational and select the minimum-cost service. Then, the incoming flow is decomposed into three subflows with intensities $\lambda_{1}, \lambda_{2}$, and $\lambda_{0}$, where $\lambda_{0}+\lambda_{1}+\lambda_{2}=\lambda$.

We assume that the costs of passengers are described by a convex combination of the price of service and the expected trip time. Without loss of generality, we define the cost of service $i$ by

$$
u_{i}(p, t)=p_{i}+\frac{c}{\mu_{i}-\lambda_{i}}, \quad i=1,2,
$$

where $p_{i}$ is the price of service $i ; 1 /\left(\mu_{i}-\lambda_{i}\right)$ is the expected trip time on the route $i ; c$ is some constant. The equilibrium intensities of the subflows $\lambda_{0}, \lambda_{1}$, and $\lambda_{2}$ satisfy the balance equation:

$$
\begin{aligned}
& p_{0}+\frac{c}{\mu_{0}-\lambda_{0}}=p_{1}+\frac{c}{\mu_{1}-\lambda_{1}}=p_{2}+\frac{c}{\mu_{2}-\lambda_{2}}, \\
& \lambda_{0}+\lambda_{1}+\lambda_{2}=\lambda,
\end{aligned}
$$

where $p_{0}$ is fixed.

Consider the pricing game with the payoff functions:

$$
\begin{aligned}
& h_{1}\left(p_{1}, p_{2}\right)=\lambda_{1} p_{1}, \\
& h_{2}\left(p_{1}, p_{2}\right)=\lambda_{2} p_{2} .
\end{aligned}
$$

Let $\lambda<\mu_{0} \leq \mu_{1} \leq \mu_{2}$ and also let $p_{2}$ be fixed. We find the best response of player 1 . To calculate a maximum of the function $h_{1}\left(p_{1}, p_{2}\right)$, consider the Lagrange function:

$$
L_{1}\left(p_{1}, \lambda_{1}, \lambda_{2}\right)=p_{1} \lambda_{1}+k_{1}\left(p_{1}+\frac{c}{\mu_{1}-\lambda_{1}}-p_{0}-\frac{c}{\mu_{0}-\left(\lambda-\lambda_{1}-\lambda_{2}\right)}\right)+k_{2}\left(p_{2}+\frac{c}{\mu_{2}-\lambda_{2}}-p_{0}-\frac{c}{\mu_{0}-\left(\lambda-\lambda_{1}-\lambda_{2}\right)}\right) .
$$

The first-order optimality conditions yield:

$$
\begin{aligned}
& \frac{\partial L_{1}}{\partial p_{1}}=\lambda_{1}+k_{1}=0 \\
& \frac{\partial L_{1}}{\partial \lambda_{1}}=p_{1}+k_{1}\left(\frac{c}{\left(\mu_{1}-\lambda_{1}\right)^{2}}+\frac{c}{\left(\mu_{0}-\lambda_{0}\right)^{2}}\right)+k_{2} \frac{c}{\left(\mu_{0}-\lambda_{0}\right)^{2}}=0, \\
& \frac{\partial L_{1}}{\partial \lambda_{2}}=k_{1} \frac{1}{\left(\mu_{0}-\lambda_{0}\right)^{2}}+k_{2}\left(\frac{1}{\left(\mu_{2}-\lambda_{2}\right)^{2}}+\frac{1}{\left(\mu_{0}-\lambda_{0}\right)^{2}}\right)=0 .
\end{aligned}
$$

Hence,

$$
\begin{aligned}
& k_{1}=-\lambda_{1}, \\
& k_{2}=\frac{\lambda_{1}\left(\mu_{2}-\lambda_{2}\right)^{2}}{\left.\left(\mu_{2}-\lambda_{2}\right)^{2}+\left(\mu_{0}-\lambda_{0}\right)^{2}\right)} .
\end{aligned}
$$

Substituting this result into (27) gives

$$
p_{1}^{*}=\lambda_{1} c\left(\frac{1}{\left(\mu_{1}-\lambda_{1}\right)^{2}}+\frac{1}{\left(\mu_{2}-\lambda_{2}\right)^{2}+\left(\mu_{0}-\lambda_{0}\right)^{2}}\right) .
$$


The best response of player 2 is obtained by analogy:

$$
p_{2}^{*}=\lambda_{2} c\left(\frac{1}{\left(\mu_{2}-\lambda_{2}\right)^{2}}+\frac{1}{\left(\mu_{1}-\lambda_{1}\right)^{2}+\left(\mu_{0}-\lambda_{0}\right)^{2}}\right) \text {. }
$$

Equations (23), (24), (29), and (30) determine the equilibrium prices and equilibrium flows for the given values $\mu_{1}$ and $\mu_{2}$.

Let us return back to the first stage of the game. At the first stage, we have the game with the payoff functions:

$$
\begin{aligned}
& H_{1}\left(\mu_{1}, \mu_{2}\right)=\lambda_{1} p_{1}^{*}-C_{1}\left(\mu_{1}\right)=\lambda_{1}^{2} c\left(\frac{1}{\left(\mu_{1}-\lambda_{1}\right)^{2}}+\frac{1}{\left(\mu_{2}-\lambda_{2}\right)^{2}+\left(\mu_{0}-\lambda_{0}\right)^{2}}\right)-C_{1}\left(\mu_{1}\right), \\
& H_{2}\left(\mu_{1}, \mu_{2}\right)=\lambda_{2} p_{2}^{*}-C_{2}\left(\mu_{2}\right)=\lambda_{2}^{2} c\left(\frac{1}{\left(\mu_{2}-\lambda_{2}\right)^{2}}+\frac{1}{\left(\mu_{1}-\lambda_{1}\right)^{2}+\left(\mu_{0}-\lambda_{0}\right)^{2}}\right)-C_{2}\left(\mu_{2}\right),
\end{aligned}
$$

where $\lambda_{1}$ and $\lambda_{2}$ satisfy (23) and (24).

5.1. Computer Simulations. Consider a passenger flow with intensity $\lambda=3$. Let the passengers choose among three possibilities for reaching a destination point: biking, using a bus, and hailing a taxi. Three capacities $\mu_{0}, \mu_{1}$, and $\mu_{2}$ correspond to these cases. For example, if $\mu_{0}=2, \mu_{1}=2.1$, and $\mu_{2}=2.5$, from equations (23), (24), (29), and (30), we find the optimal equilibrium prices:

$$
\begin{aligned}
& p_{1}^{*}=0.685, \\
& p_{2}^{*}=0.793 .
\end{aligned}
$$

The flows which correspond to these prices are

$$
\begin{aligned}
& \lambda_{0}=1.302, \\
& \lambda_{1}=0.762, \\
& \lambda_{2}=0.936 .
\end{aligned}
$$

The payoffs of the players are $h_{1}^{*}=0.5223$ and $h_{2}^{*}=0.7426$.

We assume that the costs for supporting the service of these players are described by

$$
\begin{aligned}
& c_{1}\left(\mu_{1}\right)=c_{1} \mu_{1}^{2}, \\
& c_{2}\left(\mu_{2}\right)=c_{2} \mu_{2}^{2},
\end{aligned}
$$

with $c_{1}=0.025$ and $c_{2}=0.022$. Then, the payoffs of the players calculated with the costs are

$$
\begin{aligned}
& H_{1}=0.4121, \\
& H_{2}=0.6051 .
\end{aligned}
$$

Now, for this flow $\lambda=3$, let the biking capacity be the same, $\mu_{0}=2$, and also let the players invest some money to change the capacities $\mu_{1}$ and $\mu_{2}$

We assume the first player decides to change the intensity from $\mu_{1}=2.1$ to $\mu_{1}=2.2$. In new equilibrium, we find that the optimal prices are

$$
\begin{aligned}
& p_{1}^{*}=0.669, \\
& p_{2}^{*}=0.746 .
\end{aligned}
$$

The flows which correspond to these prices are

$$
\begin{aligned}
& \lambda_{0}=1.276, \\
& \lambda_{1}=0.796, \\
& \lambda_{2}=0.927 .
\end{aligned}
$$

The payoffs of the players calculated with the costs are

$$
\begin{aligned}
& H_{1}=0.4124, \\
& H_{2}=0.5546 .
\end{aligned}
$$

Clearly, the strategy $\mu_{1}=2.2$ is more profitable for the first player than $\mu_{1}=2.1$. Applying the sequence of best responses of players, we obtain the Nash equilibrium for this case. We find the equilibrium intensities for the service:

$$
\begin{aligned}
& \mu_{1}^{*}=2.225, \\
& \mu_{2}^{*}=2.436,
\end{aligned}
$$

with the payoffs

$$
\begin{aligned}
& H_{1}^{*}=0.438, \\
& H_{2}^{*}=0.545 .
\end{aligned}
$$

Table 3 presents the resulting equilibrium $\mu_{1}^{*}$ and $\mu_{2}^{*}$ calculated for different values of the flow $\lambda=2,2.1, \ldots, 3$ and the free-of-charge flow $\mu_{0}=2$ and the costs of service in the form $c_{1}\left(\mu_{1}\right)=c_{1} \mu_{1}^{2}$ and $c_{2}\left(\mu_{2}\right)=c_{2} \mu_{2}^{2}$, where $c_{1}=0.025$ and $c_{2}=0.022$.

Clearly, for higher flows, at equilibrium, the players increase the prices of services $p_{1}^{*}$ and $p_{2}^{*}$. But the picture is not the same for the equilibrium capacities $\mu_{1}^{*}$ and $\mu_{2}^{*}$. The capacities are firstly increasing and after some instant start decreasing. The equilibrium capacity of the carrier with smaller cost is larger than the capacity with higher cost, i.e., $\mu_{2}^{*}>\mu_{1}^{*}$. Interestingly, for higher flows the difference between these capacities $\mu_{2}^{*}-\mu_{1}^{*}$ is reduced. 
TABLE 3: Optimal parameters of services in equilibrium.

\begin{tabular}{cccccccccc}
\hline$\lambda$ & $\lambda_{0}^{*}$ & $\lambda_{1}^{*}$ & $\lambda_{2}^{*}$ & $\mu_{1}^{*}$ & $\mu_{2}^{*}$ & $p_{1}^{*}$ & $p_{2}^{*}$ & $H_{1}$ & $H_{2}$ \\
\hline 2.0 & 0.822 & 0.478 & 0.701 & 2.168 & 2.681 & 0.257 & 0.344 & 0.005 & 0.083 \\
2.1 & 0.843 & 0.529 & 0.728 & 2.245 & 2.700 & 0.281 & 0.357 & 0.023 & 0.099 \\
2.2 & 0.868 & 0.577 & 0.755 & 2.307 & 2.716 & 0.305 & 0.373 & 0.043 & 0.120 \\
2.3 & 0.896 & 0.620 & 0.784 & 2.356 & 2.731 & 0.330 & 0.392 & 0.066 & 0.143 \\
2.4 & 0.928 & 0.660 & 0.812 & 2.393 & 2.740 & 0.356 & 0.414 & 0.092 & 0.171 \\
2.5 & 0.963 & 0.698 & 0.839 & 2.420 & 2.744 & 0.384 & 0.439 & 0.121 & 0.203 \\
2.6 & 1.003 & 0.730 & 0.866 & 2.430 & 2.740 & 0.415 & 0.470 & 0.156 & 0.242 \\
2.7 & 1.047 & 0.765 & 0.888 & 2.440 & 2.720 & 0.452 & 0.504 & 0.197 & 0.284 \\
2.8 & 1.102 & 0.792 & 0.906 & 2.420 & 2.680 & 0.499 & 0.550 & 0.249 & 0.340 \\
2.9 & 1.171 & 0.812 & 0.917 & 2.370 & 2.610 & 0.563 & 0.615 & 0.317 & 0.414 \\
3.0 & 1.282 & 0.813 & 0.905 & 2.230 & 2.440 & 0.688 & 0.742 & 0.435 & 0.541 \\
\hline
\end{tabular}

\section{Conclusion}

The central point in the model is the concept of rational consumers. It has been assumed that after announcing prices of services, the consumers select the minimum-cost service in the expected sense. The cost includes the price of service and also the expected trip time. It has been assumed that the consumers consider two criteria and make a decision taking both criteria into account. For example, if the incoming flow $\lambda$ is decomposed into subflows $\lambda_{i}, i \in N$, the passengers joining the flow $\lambda_{i}$ will pay $p_{i}$ for this service and waste some time $f_{i}\left(\lambda_{i}\right), i \in N$.

In future research we plan to consider the role of boundedly rational consumers in the sense of being oblivious to other consumers' influences on traffic flows, in a competitive transport system, where prices motivate the equilibrium between all players in the market, firms, and consumers. In this approach while the rational users follow a strategy that minimizes their individual cost, an oblivious user prefers fast routes.

A quite appropriate hypothesis is that the choice of consumers is balanced by an objective function. In this paper, the convolution of criteria has been adopted. It is possible to use other approaches as well. For multicriteria problems, the Nash Bargaining Solution (NBS) approach is widespread. In this case, NBS corresponds to an optimal allocation $\lambda_{i}, i \in N$, that minimizes an objective function. This issue will be considered in further research.

\section{Data Availability}

All data used can be obtained from the sources throughout the paper.

\section{Conflicts of Interest}

The authors declare that they have no conflicts of interest.

\section{References}

[1] J. G. Wardrop, "Road paper. Some theoretical aspects of road traffic research," Proceedings of the Institution of Civil Engineers, vol. 1, no. 3, pp. 325-362, 1952.

[2] J. R. Correa and N. E. Stier-Moses, "Wardrop equilibria," in Wiley Encyclopedia of Operations Research and Management Science, pp. 1-12, Springer, Berlin, Germany, 2011.

[3] Y. Sheffy, Urban Transportation Networks: Equilibrium Analysis with Mathematical Programming, Methods PrenticeHall, Englewood Cliffs, NJ, USA, 1985.

[4] V. V. Mazalov and A. V. Melnik, "Equilibrium prices and flows in the passenger traffic problem J," International Game Theory Review, vol. 18, pp. 1-19, 2016.

[5] G. Karakostas, T. Kim, A. Viglas, and H. Xia, "On the degradation of performance for traffic networks with oblivious users," Transportation Research Part B: Methodological, vol. 45, no. 2, pp. 364-371, 2011.

[6] W. Ford, J. W. Lien, V. V. Mazalov, and J. Zheng, "Riding to wall street: determinants of commute time using citi bike," International Journal of Logistics Research and Applications, vol. 22, no. 5, pp. 473-490, 2019.

[7] R. Hassin and M. Haviv, To Queue or Not to Queue. Equilibrium Behavior in Queueing Systems, Springer, Berlin, Germany, 2003.

[8] Y. A. Korillis, A. A. Lazar, and A. Orda, "Capacity allocation under noncooperative routing," Journal of IEEE Transactions on Automatic Control, vol. 42, pp. 309-325, 1997.

[9] I. Luski, "On partial equilibrium in a queuing system with two servers," The Review of Economic Studies, vol. 43, no. 3, pp. 519-525, 1976.

[10] D. Levhari and I. Luski, "Duopoly pricing and waiting lines," European Economic Review, vol. 11, no. 1, pp. 17-35, 1978.

[11] T. Roughgarden, "The price of anarchy is independent of the network topology," Journal of Computer and System Sciences, vol. 67, no. 2, pp. 341-364, 2003.

[12] US Bureau of Public Roads, Traffic Assignment Manual, US Department of Commerce, Washington, DC, USA, 1964.

[13] E. Koutsoupias and C. Papadimitriou, "Worst-case equilibria," STACS 99, vol. 1563, pp. 404-413, 1999.

[14] A. Orda, R. Rom, and N. Shimkin, "Competitive routing in multiuser communication networks," IEEE/ACM Transactions on Networking, vol. 1, no. 5, pp. 510-521, 1993.

[15] J. W. Lien, V. V. Mazalov, A. V. Melnik, and J. Zheng, "Wardrop equilibrium for networks with the BPR latency function," in Lecture Notes in Computer Science, vol. 9869, pp. 37-49, Springer, Berlin, Germany, 2016.

[16] A. A. Kulkarni and U. V. Shanbhag, "A shared-constraint approach to multi-leader multi-follower games," Set-Valued and Variational Analysis, vol. 22, no. 4, pp. 691-720, 2014.

[17] A. A. Kulkarni and U. V. Shanbhag, "An existence result for hierarchical Stackelberg v/s Stackelberg games," IEEE Transactions on Automatic Control, vol. 60, no. 12, pp. 3379-3384, 2015.

\section{Acknowledgments}

This research was supported by the Shandong Province

"Double-Hundred Talent Plan" (no. WST2017009). 